\title{
Effect of Sensor Directionality on Photoacoustic Imaging: A Study Using the k-Wave Toolbox
}

\author{
B.T. Cox and B.E. Treeby \\ Department of Medical Physics and Bioengineering, University College London, \\ Gower Street, London WC1E 6BT, UK \\ www.medphys.ucl.ac.uk/research/mle, www.k-wave.org
}

\begin{abstract}
Most image reconstruction algorithms for biomedical photoacoustic tomography make the assumption that the optically-generated ultrasonic waves are recorded by pressure detectors with an omni-directional response. In other words, the detectors are assumed to sample the pressure field exactly at a point. In practice this is rarely the case as real detectors have a finite size and often respond not purely to pressure changes but to some combination of acoustic pressure and pressure gradient (or other derivatives). This can make them less sensitive to pressure waves at some angles. The effect of this sensor directionality on photoacoustic tomography was considered here for the case of time-reversal image reconstruction. The ultrasound simulation toolbox k-Wave was used to perform the study.
\end{abstract}

Keywords: directionality, directivity, numerical acoustics, photoacoustic tomography

\section{INTRODUCTION}

Most mathematical formulations of the problem of image reconstruction in photoacoustic tomography assume that the acoustic pressure fluctuations have been measured by a set of omni-directional point pressure detectors that form a continuous and complete measurement surface around the object of interest. (Omni-directional means the detectors are equally sensitive to pressure waves from any direction.) In practice, several of these assumptions are violated. First, the measurement surface is rarely complete, i.e., it does not completely surround the object (curved detector arrays) or cover a complete plane (planar detector arrays). Second, the measurement surface is rarely continuous, i.e., the detector positions are separated in space such that the field is only sparsely sampled. Third, the detectors seldom have an omni-directional response, often exhibiting greater sensitivity to incoming waves from one particular direction.

Simple examples demonstrating the first two of these problems, and possible ways to tackle them, were given by Treeby \& Cox. ${ }^{1}$ In the current paper, the effect of detector directionality on a photoacoustic image is investigated. The study utilises the wave propagation and modelling tools within the k-Wave Toolbox (see Section 2) for simulating the data and performing time reversal image reconstruction.

\section{MODELLING ULTRASOUND WITH THE K-WAVE TOOLBOX}

k-Wave is a freely available 3rd-party toolbox for MATLAB (Mathworks, Inc., Natick, MA) which can be downloaded from http://www.k-wave.org. It uses a $k$-space pseudo-spectral solution method for the timedomain simulation of ultrasound and photoacoustic wave propagation. This section gives a brief outline of the mathematical model behind the code (further details can be found in the references). ${ }^{1-5}$

Send correspondence to B.T. Cox. bencox@mpb.ucl.ac.uk 


\subsection{Coupled Linear Acoustic Equations}

When modelling linear sound propagation in soft biological tissue, it is usually assumed that the propagation medium is isotropic, there is no net flow, and that shear waves can be neglected. Under these conditions the acoustic pressure, $p$, acoustic particle velocity, $\mathbf{u}$, and acoustic density, $\rho$, can be related by three firstorder equations corresponding to momentum conservation (sometimes called the equation of motion or Euler's equation but essentially Newton's second law), mass conservation (sometimes called the continuity equation), and an equation of state:

$$
\begin{aligned}
\frac{\partial \mathbf{u}}{\partial t} & =-\frac{1}{\rho_{0}} \nabla p, \quad \frac{\partial \rho}{\partial t}=-\rho_{0} \nabla \cdot \mathbf{u}, \\
p & =c^{2} \rho+\text { absorption terms. }
\end{aligned}
$$

These can be rearranged (without the absorption term) to give the well-known linear acoustic wave equation for heterogeneous media:

$$
\left(\rho_{0} \nabla \cdot\left(\frac{1}{\rho_{0}} \nabla\right)-\frac{1}{c^{2}} \frac{\partial^{2}}{\partial t^{2}}\right) p=0 .
$$

Here the sound speed, $c$, and ambient density, $\rho_{0}$, can vary with position $\mathbf{x}$, and the pressure, $p$, will depend in general on both position and time, $p(\mathbf{x}, t)$.

In $\mathrm{k}$-Wave, discretised versions of these three coupled first-order wave equations are solved sequentially to compute the evolution of the pressure field at each time step. The acoustic quantities are transformed into the wavenumber domain or $k$-space

$$
p(\mathbf{x}, t) \rightarrow p(\mathbf{k}, t), \quad \mathbf{u}(\mathbf{x}, t) \rightarrow \mathbf{u}(\mathbf{k}, t), \quad \rho(\mathbf{x}, t) \rightarrow \rho(\mathbf{k}, t)
$$

where the calculations are largely performed. By exploiting the speed of the Fast Fourier Transform (FFT) and a $k$-space pseudo-spectral solution method, it is possible to run efficient and accurate numerical simulations in acoustically heterogeneous media.

\subsection{Sensor Directivity}

A single frequency, unit amplitude, plane wave propagating in a direction $\mathbf{k}=\left(k_{x}, k_{z}\right)$ may be written as

$$
p(\mathbf{k}, t)=e^{i\left(k_{x} x+k_{z} z-\omega t\right)}
$$

where $\mathbf{x}=(x, z)$ and $\omega$ is its temporal frequency. (A 2D example will be given here but a similar approach holds in 3D.) If this wave is detected by a single sensor element of length $X$ which is aligned with the $x$-axis $(z=0)$, and which detects the average value of the pressure over its surface, the output of the sensor, $D(\mathbf{k})$, will be given by:

$$
\text { sensor output }=\frac{1}{X} \int_{-X / 2}^{X / 2} e^{i\left(k_{x} x-\omega t\right)} d x=D\left(k_{x}\right) e^{-i \omega t}
$$

where

$$
D\left(k_{x}\right)=\frac{1}{X} \int_{-X / 2}^{X / 2} e^{i k_{x} x} d x=\left(\frac{e^{i k_{x} X / 2}-e^{-i k_{x} X / 2}}{i k_{x} X}\right)=\operatorname{sinc}\left(k_{x} X / 2\right)
$$

The term $D\left(k_{x}\right)$ is the directionality of this sensor. (In $3 \mathrm{D}$ the sinc function becomes a Bessel function.) 


\subsection{1. k-Wave Directivity Example}

This type of directionality - which arises due to spatial averaging effects - is incorporated in k-Wave through multiplication of the sensor directionality (in this case a sinc function) with the pressure in the $k$-space domain. This is demonstrated here with a simple example. A photoacoustically generated plane wave is propagated towards a line of 31 directional sensor elements, each with the direction of maximum sensitivity set in a different direction, as illustrated in Fig. 1. The maximum output detected by each of the sensors is shown in Fig. 2A as a function of the directionality angle. It is clear that the central elements, whose directivity is maximum in the direction of the incoming wave, produce the largest response to the photoacoustic wave. The photoacoustic wave is broadband, and so the response is effectively a summation over all the frequencies in the pulse. To show that the detected wave does correspond to the sinc function derived above, the same example was repeated with the photoacoustic source replaced by a single-frequency, time-varying line source. The maximum steady state responses of the sensor elements are plotted against directivity angle, as before, in Fig. 2B. Also plotted (as the solid line) is the analytical directivity function $D$, confirming that $\mathrm{k}$-Wave is incorporating the detector directivity function accurately. Note the directivity is plotted as a function of directivity angle, $\theta$, rather than $k_{x}$. They are related by $k_{x}=|k| \sin \theta$.

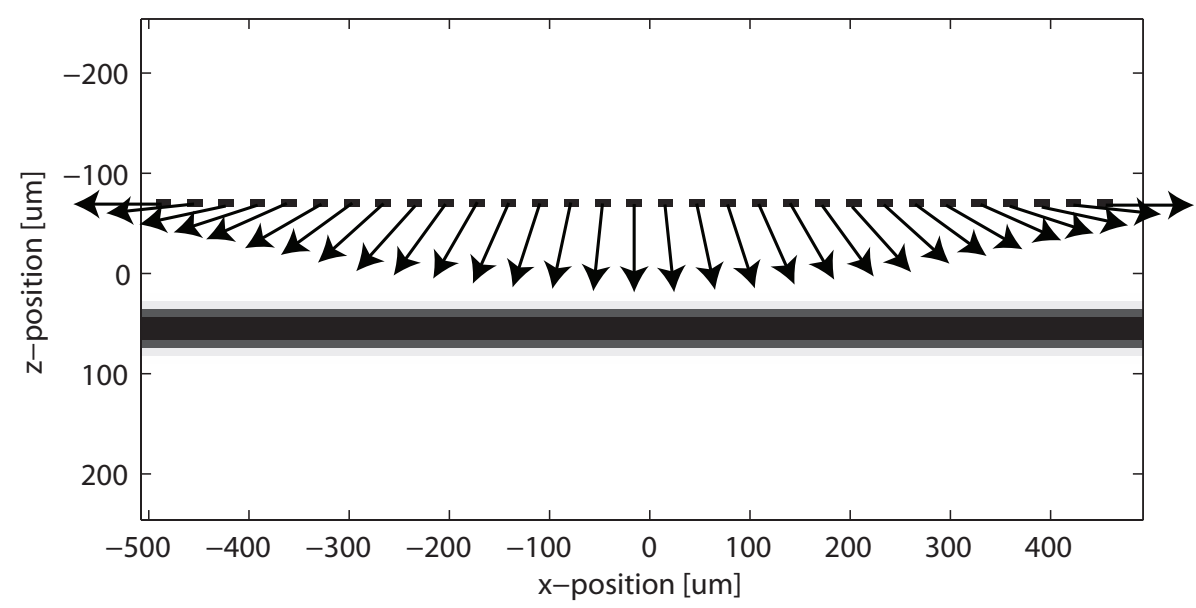

Figure 1. In this example, a line of directional sensor elements each with a different direction of maximum sensitivity (shown with the arrows) is used to detect an incoming photoacoustically generated plane wave.

\section{IMAGE RECONSTRUCTION WITH DIRECTIONAL DETECTORS}

The previous sections showed that sensor directivity can be modelled accurately in a k-space model. This now allows us to study the effect of sensor directivity on photoacoustic images. The forward and inverse (imaging) problems of photoacoustics are described briefly, followed by a demonstration of the effect of using directional detectors.

\subsection{Photoacoustic Initial Value Problem}

The forward problem in photoacoustics is to calculate the acoustic (ultrasonic) field as a function of time following the absorption of an optical pulse by an elastic fluid with heterogeneous optical absorption. (When $\mathrm{RF}$ radiation is used in place of optical, the term thermoacoustic is often preferred.) If, by absorbing the optical pulse, a region of fluid is heated sufficiently quickly to generate a local pressure increase, then an acoustic wave will result. When the heating occurs on a time-scale much shorter than the time necessary for the tissue to relax (for the density to change), the forward problem reduces to an initial value problem (IVP), with initial conditions $^{4}$

$$
\left.p(\mathbf{x}, t)\right|_{t=0}=p_{0}(\mathbf{x}),\left.\quad \mathbf{u}(\mathbf{x}, t)\right|_{t=0}=0,\left.\quad \rho(\mathbf{x}, t)\right|_{t=0}=0,
$$

where the pressure distribution at time $t=0$ is called the initial (acoustic) pressure distribution. Exact solutions to this IVP are known only in simple cases, so in general it is necessary to seek a numerical solution. 

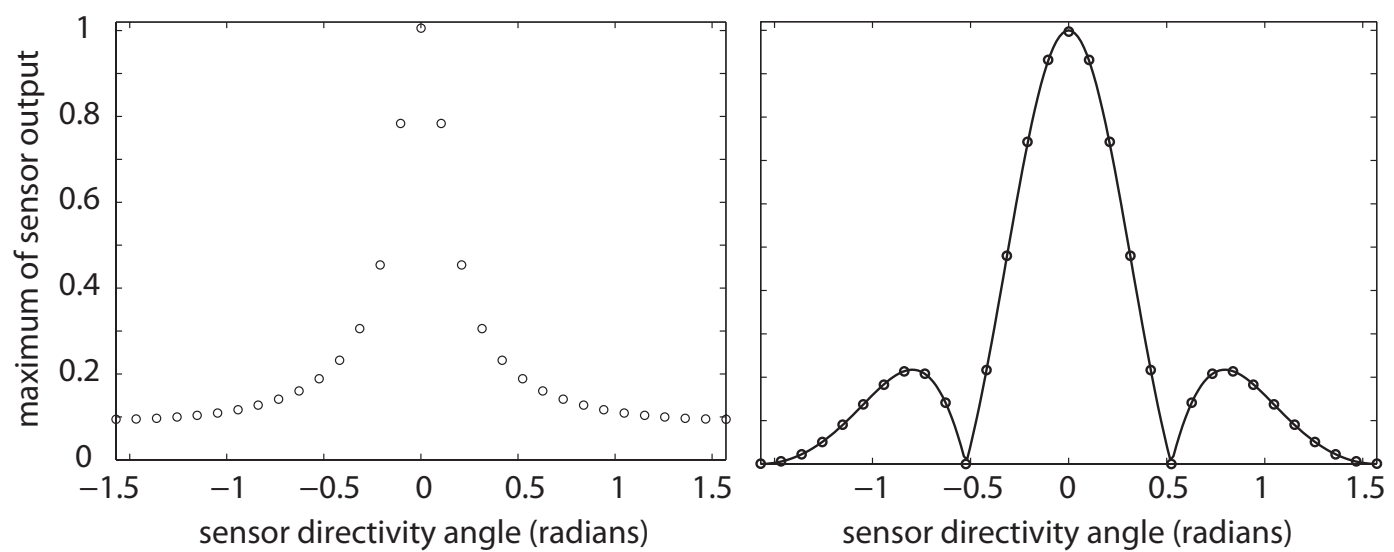

Figure 2. (A) Photoacoustic plane wave source: the directional response of the elements is clearly demonstrated. The central sensor elements, whose directivity is maximum in the direction of the incoming wave, produce the largest response to the photoacoustic wave. (B) Single-frequency plane wave source: the maximum steady state responses of the sensor elements (circles) to a single-frequency wave are compared to the directivity function $D$ (solid line), and show that k-Wave correctly incorporates this type of directivity.

\subsection{Time Reversal Image Reconstruction}

The image reconstruction problem in photoacoustic imaging is an acoustic inverse source problem: estimate the initial pressure distribution (or photoacoustic source region) given a set of acoustic pressure time histories recorded on a measurement surface. A number of algorithms have been proposed to solve this problem; here time-reversal is used. ${ }^{6-8}$ In time-reversal image reconstruction, the forward model (which is symmetrical with respect to time, in the absence of acoustic absorption) is used with the measured acoustic pressure time histories imposed as a time varying Dirichlet boundary condition at the position of the detectors on the measurement surface. When the measurement surface completely encloses the initial pressure distribution - and the detectors are omni-directional - this procedure will rebuild the acoustic pressure field inside the measurement surface in time-reversed order. When this is continued back to time $t=0$, the resulting pressure field will be the initial pressure distribution - the required photoacoustic image. This paper is interested in what happens when the detectors are not omni-directional but have greater sensitivity in one particular direction.

\subsection{Effect of Directional Detectors on Reconstructed Images}

Is sensor directivity something that should be taken into account when reconstructing photoacoustic images? What sort of artefacts might arise if it is ignored? Here, a simple 2D example provides a preliminary investigation into these questions. The photoacoustic source, given by two filled discs with a pressure magnitude (in arbitrary units) of 5 , is shown in the upper panel of Fig. 3. The resulting acoustic pressure time series recorded by a rectangular measurement surface were then simulated using $\mathrm{k}$-Wave. In this example the exterior of the rectangular measurement surface was 21.6 by $8.8 \mathrm{~mm}$ divided into 216 by 88 single pixel elements along each edge. A complete measurement surface was used so that it would be clear that any artefacts were as a result of the detector directionality and not any other factor. The direction of maximum sensitivity (or directivity angle) for each sensor element was set perpendicular to the measurement surface at that point, i.e., pointing into the domain. The corresponding equivalent sensor element size, $X$, was set to $2 \mathrm{~mm}$. The resulting image reconstructed using time reversal image reconstruction with the time series recorded by the directional detectors is shown in the lower panel in Fig. 3. The circular discs have taken on a square appearance with artefacts trailing away in each direction. An $x$-profile through the centre of the larger disc is shown in Fig. 4 for comparison. Despite having a complete measurement surface, the directionality of the sensor elements has introduced noticeable banding artefacts along the directions of maximum sensitivity. 

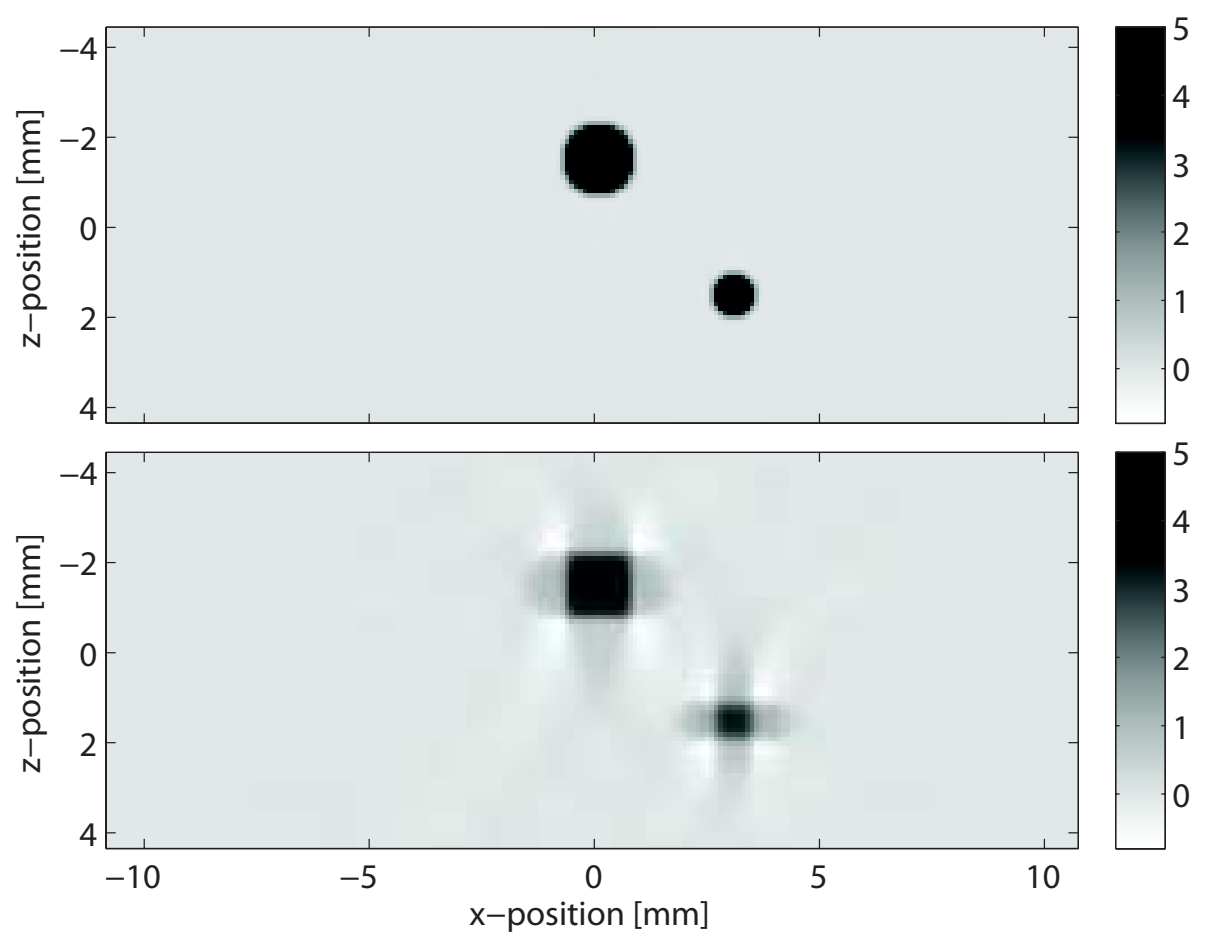

Figure 3. Two-dimensional example illustrating the effect of sensor directivity on time reversal image reconstruction. The upper panel illustrates the initial photoacoustic pressure distribution used to simulate the acoustic pressure time series recorded over a rectangular measurement surface around the outer edge of the displayed region. The directivity angle of each sensor element is set perpendicular to the measurement surface. The lower panel illustrates the corresponding time reversal image reconstruction. Despite having a continuous and complete measurement surface, the reconstruction displays artefacts due to the sensor directivity.

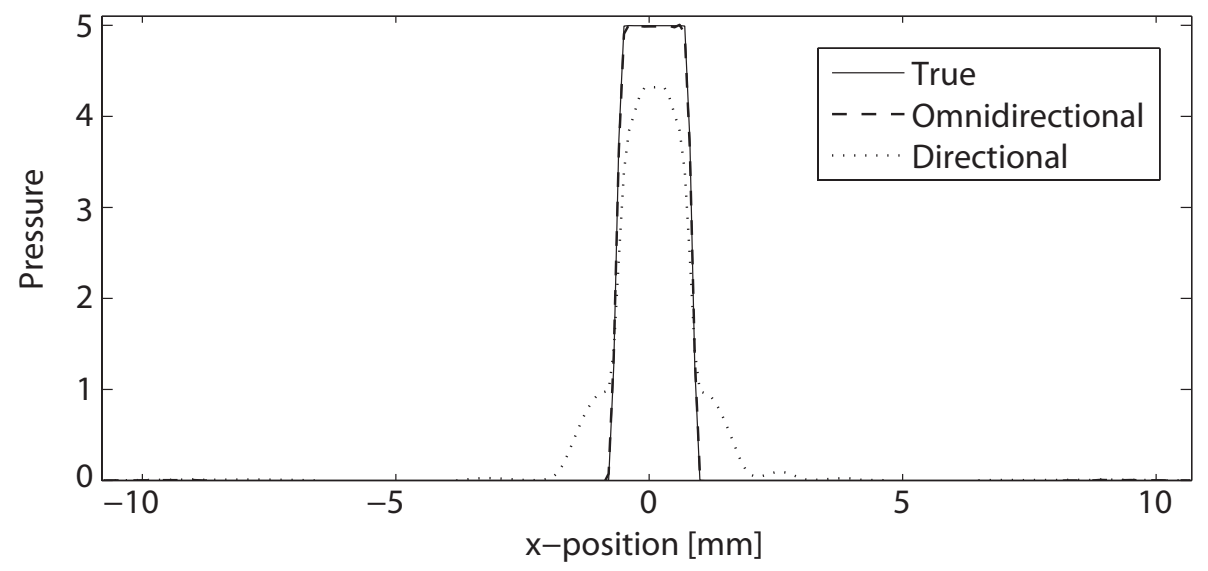

Figure 4. $x$-profile through the larger disc shown in Fig. 3 highlighting the reconstruction artefacts due to sensor directivity. 


\section{CONCLUSIONS}

Most image reconstruction algorithms for biomedical photoacoustic tomography make the assumption that the optically-generated ultrasonic waves are recorded by pressure detectors with an omni-directional response. In practice this is rarely the case as real detectors have a finite size and often respond to some combination of acoustic pressure and pressure gradient (or other derivatives) and not purely to pressure changes. This can make them less sensitive to pressure waves at some angles. Here, the theoretical directivity that results from a sensor element in $2 \mathrm{D}$ recording the average pressure value over its surface was derived. This was numerically implemented in $k$-space within the $\mathrm{k}$-Wave modelling toolbox. The effect of sensor directionality on photoacoustic tomography was then investigated for the case of time-reversal image reconstruction. Even when a complete and continuous measurement surface was used, the inclusion of sensor directivity introduces image reconstruction artefacts. For the example investigated here, these artefacts appear to follow the direction of maximum sensitivity of the rectangular faces of the measurement surface. This finding has important ramifications both for the structural fidelity and quantitative accuracy of images reconstructed from experimental data, as most practical sensor elements arrays exhibit some directionality.

\section{ACKNOWLEDGMENTS}

The authors would like to thank Paul Beard for helpful discussions. This work was supported by the Engineering and Physical Sciences Research Council, UK.

\section{REFERENCES}

1. B. E. Treeby and B. T. Cox, "k-Wave: A MATLAB toolbox for the simulation and reconstruction of photoacoustic wave-fields," J. Biomed. Opt. , In Press, 2010.

2. M. Tabei, T. D. Mast, and R. C. Waag, "A k-space method for coupled first-order acoustic propagation equations," J. Acoust. Soc. Am. 111(1), pp. 53-63, 2002.

3. B. T. Cox, S. Kara, S. R. Arridge, and P. C. Beard, "k-space propagation models for acoustically heterogeneous media: Application to biomedical photoacoustics," J. Acoust. Soc. Am. 121(6), pp. 3453-3464, 2007.

4. B. T. Cox and P. C. Beard, "Modeling photoacoustic propagation in tissue using k-space techniques," in Photoacoustic Imaging and Spectroscopy, L. V. Wang, ed., pp. 25-34, CRC Press, London, 2009.

5. J. C. Tillett, M. I. Daoud, J. C. Lacefield, and R. C. Waag, "A k-space method for acoustic propagation using coupled first-order equations in three dimensions," J. Acoust. Soc. Am. 126(3), pp. 1231-1244, 2009.

6. Y. Xu and L. V. Wang, "Time reversal and its application to tomography with diffracting sources," Phys. Rev. Lett. 92(3), p. 033902, 2004.

7. P. Burgholzer, G. J. Matt, M. Haltmeier, and G. Paltauf, "Exact and approximative imaging methods for photoacoustic tomography using an arbitrary detection surface," Phys. Rev. E 75(4), p. 046706, 2007.

8. B. T. Cox and B. E. Treeby, "Artifact trapping during time reversal photoacoustic imaging for acoustically heterogeneous media," IEEE Trans. Med. Imaging, In Press, 2010. 